\title{
Activation Energy of Local Polymer Motions Estimated from the Fluorescence Depolarization Measurements
}

\author{
Shunsuke Yokotsuka, Yasuo OKada, Yasuhisa Tojo, \\ Takashi SASAKI, and Masahide YAMAMOTO* \\ Department of Polymer Chemistry, Kyoto University, Kyoto 606, Japan
}

(Received September 4, 1990)

\begin{abstract}
Activation energies of local conformational transitions in polymers were investigated by the fluorescence depolarization method. The mean relaxation time related to the local conformational transitions was measured for dilute solutions of the anthracene-labeled polystyrene (PS), and anthracene-labeled poly $(\alpha$-methylstyrene) (P $\alpha \mathrm{MS})$ in various solvents. The obtained data were successfully analyzed by use of the generalized formula of Helfand based on the reaction rate theory of Kramers. The results showed that the activation energy is larger in poor solvents than in good solvents which reflects the chain expansion effect. It is clear that the apparent activation energy is influenced by two types of polymer-solvent interactions, i.e., the frictional interaction (short-range interaction) and the chain expansion effect (long range interaction), and that the former can also be evaluated roughly by the present analysis.
\end{abstract}

KEY WORDS Activation Energy / Conformational Transition / Local Motions / Fluorescence Depolarization / Anthracene-Labeled Polystyrene / Anthracene-Labeled Poly ( $\alpha$-methylstyrene) /

Local motions of flexible polymer chains can be understood as the transition between the stable conformational states (conformational transition). Such transition occurs passing through the activated (transient) state with a certain barrier height $E_{\mathrm{a}}$ (activation energy), and $E_{\mathrm{a}}$ is essentially inherent to individual polymer chains. However, the experimental values reported hitherto for the same polymer chain are inconsistent. ${ }^{1,2}$ The results in dilute polymer solutions showed that $E_{\text {a }}$ depends on the solvent (viscosity and compatibility with the polymer), experimental technique, and occasionally on temperature. The values of $E_{\mathrm{a}}$ observed experimentally in dilute polymer solutions are influenced by at least four factors, i.e., (A) barrier height inherent to the individual polymer chain (intrinsic activation energy), (B) exerted friction in the course of conformational transition, (C) local density of polymer segment which depends on chain expansion, and (D) observing motional scale. (B) and (C) are concerned with the interaction between the polymer segments and solvent molecules in short and long range, respectively. The factor (D) may be dependent merely on the experimental method.

This study was focused on the first three factors, (A), (B), and (C), to investigate the effect of three factors on $E_{\mathrm{a}}$, especially to treat the effect of (B), i.e., friction between the polymer segment and the solvent molecules, on the basis of the generalized treatment of conformational trasitions in polymers. ${ }^{3}$

The concept of the activation energy of local motions in polymers was proposed by Helfand ${ }^{3}$ which was based on the reaction rate theory of Kramers. ${ }^{4}$ In this theory, a rather simplified model is treated as a double-welled potential, and expressions for the temperature dependence of the conformational transition rate is formulated, which contain the friction factor

\footnotetext{
* To whom all correspondence should be addressed.
} 
with the solvent. The experimental observation of the 'solvent dependence' of $E_{\mathrm{a}}$ may be partially due to the difference in the manner of frictional interaction between the polymer segments and the solvent. This difference corresponds to the effect of (B) (friction between the polymer segment and solvent molecules).

Here, we employ the fluorescence depolarization method for the estimation of local relaxation time. The sample polymers were labeled with anthracene in the middle of the polymer chain. Such a method has been utilized for the study of local polymer chain dynamics. ${ }^{5,6}$ Now, this technique is suitable for the present purpose, because it allows us to observe only the main chain motions directly. In this paper, we treat polystyrene (PS) of which the activation energy of local motions has been investigated rather widely. ${ }^{1,7-13}$ For comparison, we will also treat anthracene-labeled $\operatorname{poly}(\alpha$-methylstyrene) (P $\alpha \mathrm{MS})$.

\section{EXPERIMENTAL}

The anthracene-labeled samples were synthesized by the anionic polymerization and succeeding coupling reaction by 9,10 -bis(bromomethyl)anthracene. Synthesis of the anthracene-labeled PS was executed as follows. First, anionic polymerization of styrene was initiated by $n$-butyllithium in benzene at $7^{\circ} \mathrm{C}$. The polymerization reaction was executed at $7^{\circ} \mathrm{C}$ until the whole monorners were exhausted. Thereafter, a small amount of the coupling reagent 9,10-bis(bromomethyl)anthracene which had been dissolved in THF, was added to the reaction vessel at $7^{\circ} \mathrm{C}$ with constant stirring. The solution was stirred for several hours at $7^{\circ} \mathrm{C}$ until the reaction was completed. The reacted solution was poured into a large amount of methanol, and the precipitated polymer was separated. The polymer was purified by reprecipitation in methanol. The anthracene-labeled $\mathrm{P} \alpha \mathrm{MS}$ was synthesized in a manner similar to that mentioned above. The anionic polymerization of $\alpha$-methylstyrene was executed with $n$-butyllithium in THF at $-78^{\circ} \mathrm{C}$. After stirring was continued for $1 \mathrm{~h}$ at $-78^{\circ} \mathrm{C}$, the temperature was raised to room temperature, and the solution was stirred for $1 \mathrm{~h}$ for depolymerization. Thereafter, the solution was again cooled to $-78^{\circ} \mathrm{C}$, and was stirred for $1 \mathrm{~h}$ for repolymerization. Finally, the coupling reagent 9,10-bis(bromomethyl)anthracene was added, and the solution was stirred for $c a .5 \mathrm{~h}$ at $-78^{\circ} \mathrm{C}$. The polymer was separated and purified by precipitation in methanol. Furthermore, P $\alpha$ MS had to be purified by fractionation by GPC to eliminate the end-labeled fraction.

The above obtained sample polymers were characterized by GPC and ${ }^{13} \mathrm{C}-\mathrm{NMR}$, and as a result, the molecular weight and tacticity was determined as shown in Table I.

Time-resolving measurements of fluorescence depolarization were done for dilute solutions of butyl acetate, cyclohexane, dioxane, cyclohexanone, and tripropionin (glycerol tripropionate). $\mathrm{P} \alpha \mathrm{MS}$ is insoluble in butyl acetate and in tripropionin. Table II shows the viscosity and its activation energy of these solvents. Intrinsic viscosity $[\eta]$ was also measured at $34.5^{\circ} \mathrm{C}$ as shown in Table III (cyclohexane is a theta solvent for both PS and

Table I. Molecular weight and triad tacticity of the samples

\begin{tabular}{lcc}
\hline & PS & P $\alpha \mathrm{MS}$ \\
\hline$M_{n} \times 10^{-4}$ & 9.2 & 20.5 \\
$M_{w} \times 10^{-4}$ & 9.7 & 21.5 \\
$M_{w} / M_{n}$ & 1.05 & 1.05 \\
$m m: m r: r r$ & $22: 48: 30$ & $8: 50: 42$ \\
\hline
\end{tabular}

Table II. Solvent viscosity $\eta$ and its activation energy

\begin{tabular}{lcc}
\hline & $\eta$ at $20^{\circ} \mathrm{C} / \mathrm{cP}$ & $E_{\eta} / \mathrm{kcal} \mathrm{mol}^{-1}$ \\
\hline Butyl acetate & 0.740 & 2.5 \\
Cyclohexane & 0.979 & 2.9 \\
Dioxane & 1.313 & 3.0 \\
Cyclohexanone & 2.0 & 3.3 \\
Tripropionin & 6.1 & 6.5 \\
\hline
\end{tabular}


Table III. Intrinsic viscosity at $34.5^{\circ} \mathrm{C}$

\begin{tabular}{llllll}
\hline & \multicolumn{2}{c}{$\mathrm{PS}$} & & \multicolumn{2}{c}{$\mathrm{P} \alpha \mathrm{MS}$} \\
\cline { 2 - 3 } & {$[\eta] / \mathrm{dlg}^{-1}$} & {$[\eta] /[\eta]_{\theta}$} & {$[\eta] / \mathrm{dl} \mathrm{g}^{-1}$} & {$[\eta] /[\eta]_{\theta}$} \\
\hline Butyl acetate & 0.347 & 1.25 & & \\
Cyclohexane & 0.277 & 1 & & 0.298 & 1 \\
Dioxane & 0.471 & 1.70 & 0.567 & 1.90 \\
Cyclohexanone & 0.433 & 1.56 & 0.589 & 1.98 \\
Tripropionin & 0.222 & 0.801 & & \\
\hline
\end{tabular}

$\mathrm{P} \alpha \mathrm{MS}$ at this temperature). Molecular weights of the samples used for the measurement of intrinsic viscosity are $M_{w}=11.6 \times 10^{4}$ for PS and $1.87 \times 10^{4}$ for $\mathrm{P} \alpha \mathrm{MS}$. Table III shows that cyclohexanone and dioxane are good solvents for PS and P $\alpha \mathrm{MS}$, while tripropionin is a poor solvent for PS. Butyl acetate is a good solvent for PS, but is poorer than dioxane and cyclohexanone.

The concentration of all the sample solutions were kept less than $0.1 \mathrm{wt} \%$ to exclude the polymer-polymer interaction as well as the migration of the electronic excited states (the chromophore concentrations were kept less than $10^{-5} \mathrm{M}$ ). All these solutions were degassed before the measurements were taken. The measurements were done by use of the nanosecond single-photon counting system in our laboratory, ${ }^{6}$ in which the temperature was controlled by circulating water from a thermo-regulated bath, or by thermo-regulated $\mathrm{N}_{2}$ gas flow.

From the time-resolved data, we estimated the fluorescence lifetime of the anthracene label. However, the decay profiles for the anthracene-labeled PS and anthracene-labeled P $\alpha$ MS exhibit nonexponential curves, which are probably due to certain interaction between the anthracene group and neighboring phenyl groups. Therefore, in these cases, the decay curves of the fluorescence emission intensity $F(t)$ had to be analyzed with triexponential function (experimental function)

$$
\begin{aligned}
F(t)= & a_{1} \exp \left(-t / \tau_{1}\right)+a_{2} \exp \left(-t / \tau_{2}\right) \\
& +a_{3} \exp \left(-t / \tau_{3}\right)
\end{aligned}
$$

Table IV. Averaged lifetime of the anthracene label

\begin{tabular}{lcc}
\hline \multicolumn{1}{c}{ Solvent } & PS $/ \mathrm{ns}$ & P $\alpha \mathrm{MS} / \mathrm{ns}$ \\
\hline Butyl acetate & $8.19\left(25.4^{\circ} \mathrm{C}\right)$ & \\
Cyclohexane & $7.58\left(26.1^{\circ} \mathrm{C}\right)$ & $7.70\left(26.2^{\circ} \mathrm{C}\right)$ \\
Dioxane & $7.70\left(26.0^{\circ} \mathrm{C}\right)$ & $6.92\left(26.0^{\circ} \mathrm{C}\right)$ \\
Cyclohexanone & $7.90\left(26.5^{\circ} \mathrm{C}\right)$ & $7.20\left(26.0^{\circ} \mathrm{C}\right)$ \\
Tripropionin & $7.69\left(25.0^{\circ} \mathrm{C}\right)$ & \\
\hline
\end{tabular}

by the method of nonlinear least-squares fitting, and the averaged lifetime $\langle\tau\rangle$ was estimated as

$$
\langle\tau\rangle=a_{1} \tau_{1}+a_{2} \tau_{2}+a_{3} \tau_{3}
$$

Table IV shows the obtained averaged lifetimes.

The time-resolved data of fluorescence anisotropy ratio $r(t)$ were analyzed by the method of nonlinear least-squares fitting as described previously. ${ }^{6}$ In this study, triexponential function (experimental function)

$$
\begin{aligned}
r(t)= & b_{1} \exp \left(-t / T_{1}\right)+b_{2} \exp \left(-t / T_{2}\right) \\
& +b_{3} \exp \left(-t / T_{3}\right)
\end{aligned}
$$

was fitted to the observed $r(t)$, and the mean relaxation time $T_{\mathrm{m}}$ defined as ${ }^{5}$

$$
T_{\mathrm{m}}=r_{0}{ }^{-1} \int_{0}^{\infty} r(t) \mathrm{d} t
$$

was estimated, which represents the effective correlation time of the local polymer relaxation. Also, $T_{m}$ is considered as a measure of the apparent chain stiffness (the dynamic chain stiffness).

\section{RESULTS AND DISCUSSION}

The mean relaxation time $T_{\mathrm{m}}$ was measured in the temperature range $-10-40^{\circ} \mathrm{C}$ for each solvent. For the evaluation of the activation energy, we first employed the usual method. ${ }^{7,11}$ We assume that the friction in the course of conformational transition is rather high (so-called the diffusion limit). In this case, we obtained the relation ${ }^{3}$

$$
T_{\mathrm{m}}=A \zeta \exp \left(E_{\mathrm{a}} / R T\right)
$$


where $\zeta$ is the friction coefficient, and $R$ the gas constant. If we employ the Stokes law that $\zeta \propto \eta$, and if the viscosity $\eta$ obeys the equation $\eta=A_{\eta} \exp \left(E_{\eta} / R T\right)$, we find ${ }^{7,11}$

$$
T_{\mathrm{m}}=A^{\prime} \exp \left[\left(E_{\eta}+E_{\mathrm{a}}\right) / R T\right]
$$

Therefore, we can obtain the activation energy of local polymer motion $E_{\mathrm{a}}$ directly from the slope of the plot $\ln \left(T_{\mathrm{m}} / \eta\right) v s .1 / T$.

First, we will discuss the results obtained for the PS chain. Figure 1 shows such plots for PS in the four solvents. Table $\mathrm{V}$ shows the obtained values of $E_{\mathrm{a}}$. Figure 1 clearly shows that the absolute value of $T_{\mathrm{m}} / \eta$ is markedly larger in the poor solvent (cyclohexane) than in the good solvents (other three solvents). The same findings have been reported by other investigators, ${ }^{7,14}$ and the explanation given

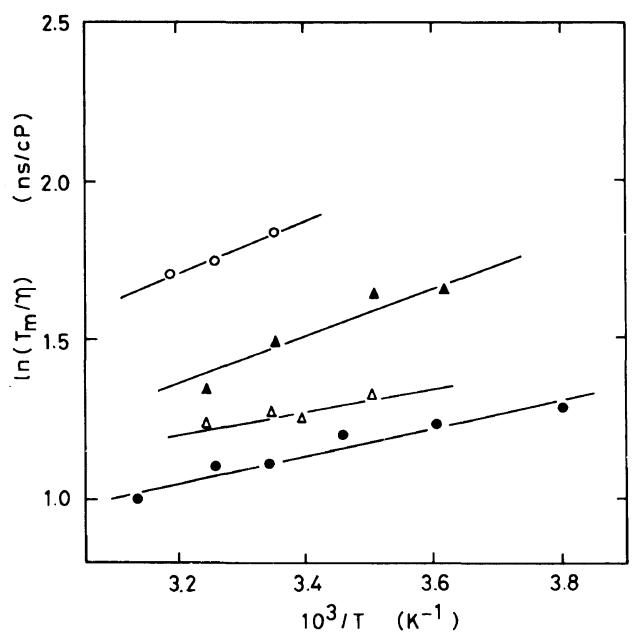

Figure 1. Plots of $T_{\mathrm{m}} / \eta$ vs. $1 / T$ for PS in dilute solutions of butyl acetate $(\boldsymbol{\Delta})$, cyclohexane $(\bigcirc)$, dioxane $(\triangle)$, and cyclohexanone (

Table V. Activation energies obtained from the plots of $\ln \left(T_{\mathrm{m}} / \eta\right)$ vs. $1 / T$

\begin{tabular}{lcc}
\hline \multicolumn{1}{c}{ Solvent } & $\mathrm{PS} / \mathrm{kcal} \mathrm{mol}^{-1}$ & $\mathrm{P} \alpha \mathrm{MS} / \mathrm{kcal} \mathrm{mol}^{-1}$ \\
\hline Butyl acetate & 1.5 & \\
Cyclohexane & 1.6 & 3.0 \\
Dioxane & 0.7 & 1.0 \\
Cyclohexanone & 0.8 & 1.1 \\
Tripropionin & -1.9 & \\
\hline
\end{tabular}

was that the local segment density becomes higher in poor solvents than in good solvents. ${ }^{14}$ Figure 2 shows the same plot for PS in tripropionin. Here, we obtained an unrealistic negative activation energy value. This indicates that the usual analysis based on eq 6 employed here is insufficient for the present data.

Now, we note one approximation in the above analysis based on eq 6 that the friction coefficient $\zeta$ is determined from the solvent viscosity. In other words, the friction factor between the polymer segment and solvent moiecule is substituted for that between solvent molecules. Our result in tripropionin shows that the friction (molecular interaction) between the polymer segment and the solvent molecule is markedly different from that between solvent molecules. Probably, the polymer-solvent interaction is rather weak in the PS-tripropionin system.

Helfand ${ }^{3}$ treated the reaction rate theory of Kramers in two limits, i.e., the diffusion limit (high friction) and non-diffusion limit (low friction). In the latter limit, $E_{\mathrm{a}}$ is directly observed without the knowledge of solvent viscosity, and in the former limit which we employed in the above analysis (eq 6), the apparent activation energy becomes the sum of $E_{\mathrm{a}}$ and $E_{\eta}$. According to Helfand, these two

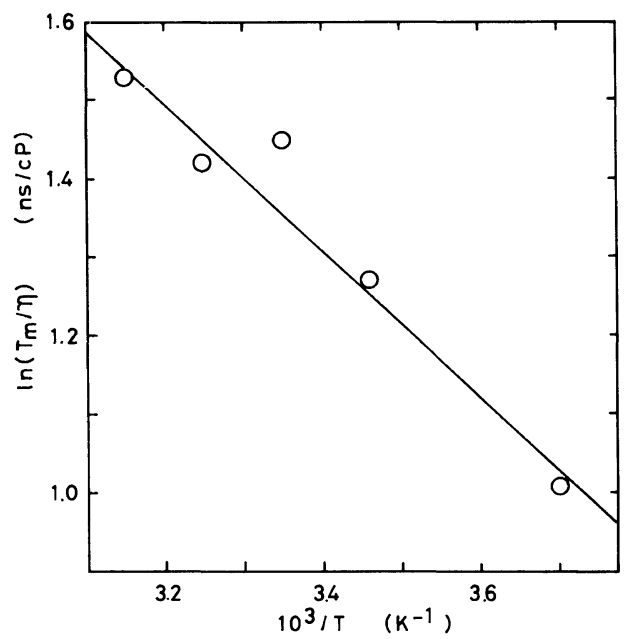

Figure 2. Plot of $T_{m} / \eta$ vs. $1 / T$ for PS in tripropionin. 
limits can be connected by the following generalized formula as ${ }^{3}$

$$
\begin{aligned}
T_{\mathrm{m}}= & \left\{1 / 2+\left[1 / 4+A_{1} \exp \left(-2 E_{\eta} / R T\right)\right]^{1 / 2}\right\} \\
& A_{2} \exp \left[\left(E_{\eta}+E_{\mathrm{a}}\right) / R T\right]
\end{aligned}
$$

where the constants $A_{1}$ and $A_{2}$ depend on the intrinsic potential profile for the conformational transition in polymer chain, on size (mass) of the motional unit of the observed motion, and also on the manner of frictional interaction between the polymer segment and solvent molecule as will be mentioned below.

If we regard the friction constant $\zeta$ appearing in Helfand's paper ${ }^{3}$ as the strength of interaction between polymer segment and solvent molecule, and if the size of the motional unit (motional mode) does not change with temperature, $A_{1}$ and $A_{2}$ depend on the solvent in the following way: (1) in the diffusion (high friction) limit, $A_{1}$ is small and $A_{2}$ is large, and the fashion of the polymer-solvent interaction is 'cooperative' or 'sticking'. (2) as the polymer-solvent interaction of friction becomes weaker, $A_{1}$ increases and $A_{2}$ decreases. (3) in the non-diffusion (low friction) limit, $A_{1}$ is large and $A_{2}$ is small, and the fashion of the polymer-solvent interaction is 'slipping'. Thus, the constants $A_{1}$ and $A_{2}$ can be regarded as a rough (qualitative) measure for the interaction strength (fashion of the frictional interaction) between polymer and solvent for the same polymer.

Now, we will apply eq 7 for the present experimental results. In the present analysis for sufficiently dilute solutions $(<0.1 \mathrm{wt} \%)$, the temperature dependence of the polymer-solvent interaction is comparable to that of solvent viscosity i.e., we suppose that $E_{\eta}$. in eq 7 is nearly equal to the activation energy of the solvent viscosity listed in Table II. Thus, we fitted eq 7 to the experimental data by the nonlinear least-squares method, treating $A_{1}$, $A_{2}$, and $E_{\mathrm{a}}$ as variable parameters. Table VI shows the obtained best-fit parameters. Figures 3 and 4 show the obtained best-fit curves.

In Table VI, the activation energy $E_{\mathrm{a}}$ for PS in tripropionin shows a positive value. Thus, the present analysis with eq 7 is successful. As Table VI shows, $E_{\mathrm{a}}$ is larger in poor solvents such as butyl acetate, cycloohexane, and tripropionin $\left(E_{\mathrm{a}}=1.6-1.9 \mathrm{kcal} \mathrm{mol}^{-1}\right)$, than in good solvents such as dioxane and cyclohexanone $\left(E_{\mathrm{a}}=1.2 \mathrm{kcal} \mathrm{mol}^{-1}\right)$. This is due to the chain expansion effect, i.e., in a poor solvent, the local segment density is large leading to

Table VI. Activation energies obtained from

\begin{tabular}{|c|c|c|c|}
\hline Solvent & & PS & $\mathrm{P} \propto \mathrm{MS}$ \\
\hline \multirow[t]{3}{*}{ Butyl acetate } & $\mathrm{A}_{1}$ & 170 & \\
\hline & $\mathrm{A}_{2}(\mathrm{~ns})$ & $2.7 \times 10^{-3}$ & \\
\hline & $\mathrm{E}_{\mathrm{a}}\left(\mathrm{kcal} \mathrm{mol}^{-1}\right)$ & 1.6 & \\
\hline \multirow[t]{3}{*}{ Cyclohexane } & $\mathrm{A}_{1}$ & 1.0 & 1.1 \\
\hline & $A_{2}(n s)$ & $2.4 \times 10^{-3}$ & $3.8 \times 10^{-4}$ \\
\hline & $\mathrm{E}_{\mathrm{a}}\left(\mathrm{kcal} \mathrm{mol}^{-1}\right)$ & 1.7 & 3.1 \\
\hline \multirow[t]{3}{*}{ Dioxane } & $A_{1}$ & $2.3 \times 10^{3}$ & $1.3 \times 10^{3}$ \\
\hline & $\mathrm{A}_{2}(\mathrm{~ns})$ & $3.4 \times 10^{-3}$ & $2.4 \times 10^{-3}$ \\
\hline & $\mathrm{E}_{\mathrm{a}}\left(\mathrm{kcal} \mathrm{mol}^{-1}\right)$ & 1.2 & 1.5 \\
\hline \multirow[t]{3}{*}{ Cyclohexanone } & $A_{1}$ & $8.5 \times 10^{3}$ & $1.1 \times 10^{5}$ \\
\hline & $\mathrm{A}_{2}(\mathrm{~ns})$ & $3.1 \times 10^{-3}$ & $2.1 \times 10^{-4}$ \\
\hline & $\mathrm{E}_{\mathrm{a}}\left(\mathrm{kcal} \mathrm{mol}^{-1}\right)$ & 1.2 & 2.5 \\
\hline \multirow[t]{3}{*}{ Tripropionin } & $\mathrm{A}_{1}$ & $5.0 \times 10^{9}$ & \\
\hline & $\mathrm{A}_{2}(\mathrm{~ns})$ & $8.0 \times 10^{-6}$ & \\
\hline & $\mathrm{E}_{\mathrm{a}}\left(\mathrm{kcal} \mathrm{mol}^{-1}\right)$ & 1.9 & \\
\hline
\end{tabular}
the analysis with eq 7

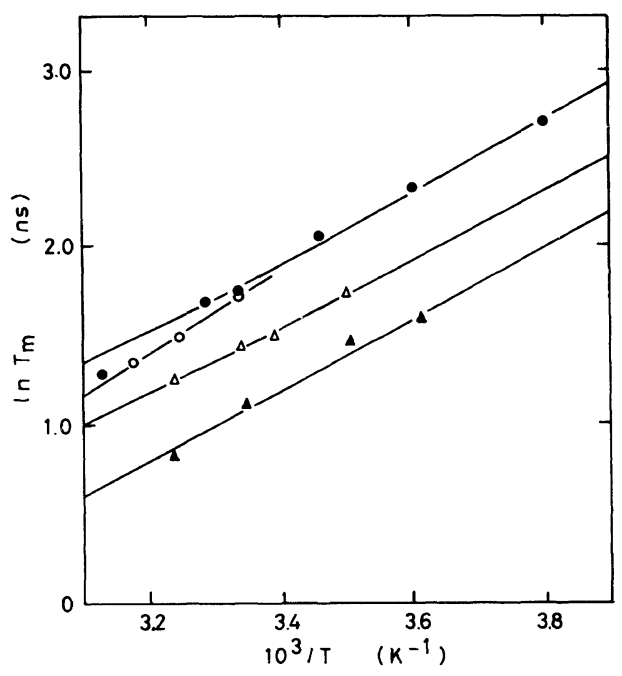

Figure 3. Plots of $T_{m} v$ s. $1 / T$ for PS. Solid lines show the result of the fitting analysis with eq 7 . Each symbol represents each solvent in the same way as in Figure 1. 


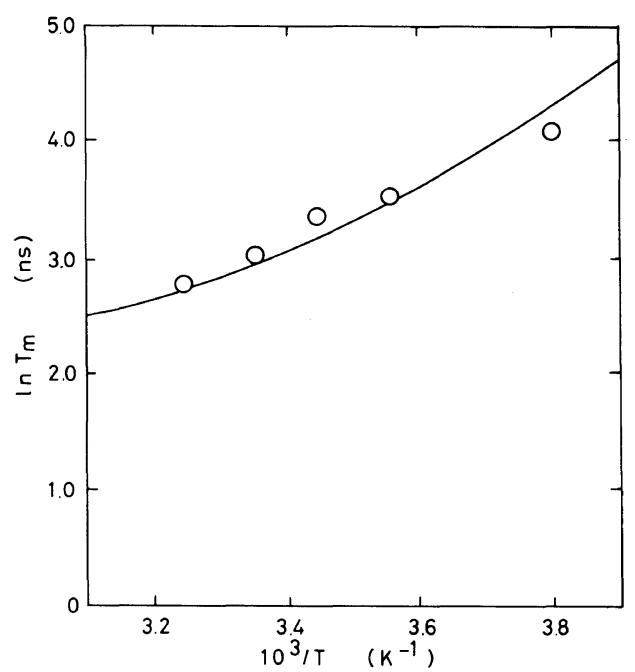

Figure 4. Result of the fitting analysis with eq 7 (solid line) for PS in tripropionin.

large barrier height for the conformational transition. $^{7}$ In addition, the temperature dependence of the chain expansion is stronger in a poor solvent than in a good solvent, and this may contribute to the stronger temperature dependence of $T_{\mathrm{m}}$ in poor solvent than in good solvent. ${ }^{14}$

Comparison of the results shown in Tables $\mathrm{V}$ and VI revealed no significant differences in $E_{\mathrm{a}}$ in the case of butyl acetate and cyclohexane. Therefore, the usual treatment based on eq 6 is generally adequate for the local motions in these two solvents, i.e., the local relaxation time $T_{\mathrm{m}}$ can be described by eq 6 (high friction limit). The values of $A_{1}$ in butyl acetate and cyclohexane also show that the polymer-solvent interaction of friction is in the sticking manner, i.e., the frictional interaction is strong. As for tripropionin, the values of $A_{1}$ and $A_{2}$ show that the polymer-solvent interaction of friction is weakest among the five solvents, even though this system does not belong to the non-diffusion (low friction) limit, i.e., the obtained $E_{\mathrm{a}}$ in Table VI does not coincide with the apparent activation energy from the plot ln $T_{\mathrm{m}} v s .1 / \mathrm{T}\left(4.6 \mathrm{kcal} \mathrm{mol}^{-1}\right)$.

It should also be noticed that the strength of the polymer-solvent interaction of friction reflected in $A_{1}$ and $A_{2}$ is independent of the chain expansion effect. For example, the $A_{1}$ for tripropionin (poor solvent) is fairly large, while the $A_{1}$ for cyclohexane (poor solvent) is small. Also, the $A_{1}$ for cyclohexanone (good solvent) is large. Thus, a strong interaction (high friction) between polymer and solvent does not always lead to large chain expansion. Indeed, the frictional interaction between polymer chain and solvent molecule is essentially independent of the chain expansion effect. The former is due to the short range interaction and based on directly local molecular dynamics, while the latter is due to the long range interaction based on thermodynamics. This finding implies that the above two interactions occur from essentially different origins or by different mechanisms. Thus, the two effects (B) and (C) mentioned in the introduction should be treated as independent ones.

Tables V and VI also show the results obtained for the P $\alpha \mathrm{MS}$ chain. Figure 5 shows the best-fit results from the analysis with eq 7 . The results obtained here qualitatively coincide with those for PS. The activation energy is larger in cyclohexane (poor solvent) than in dioxane and cyclohexanone (good solvents). The absolute values of $E_{\mathrm{a}}$ are larger than those of PS. Probably, this is mainly due to the difference in the intrinsic barrier height, i.e., this reflects the difference in the chemical structure of polymer chain [factor (A) presented in introduction]. Indeed, it is reasonable to assume that the $\alpha$-methyl group causes steric hindrance in the conformational transition, and the results of conformation energy calculations indicate the same. ${ }^{15,16} \mathrm{It}$ is also noticed that the absolute values of $T_{\mathrm{m}}$ of P $\alpha \mathrm{MS}$ are larger than those of PS. Table VII shows $T_{\mathrm{m}}$ in cyclohexanone for example. The molecular weight is larger for the $\mathrm{P} \alpha \mathrm{MS}$ sample than for PS sample employed here (see Table I), but these molecular weights are high enough to neglect the chain-end effect. ${ }^{7,9}$ 


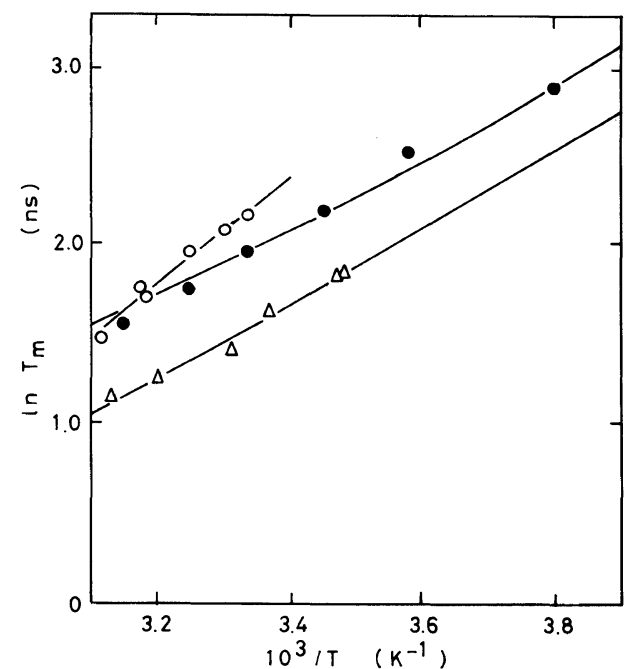

Figure 5. Results of the fitting analysis with eq 7 (solid line) for $\mathrm{P} \alpha \mathrm{MS}$ in dilute solutions of cyclohexane $(\mathrm{O})$, dioxane $(\triangle)$, and cyclohexanone

Table VII. Comparison of $T_{m}$ between PS and $\mathrm{P} \alpha \mathrm{MS}$ in cyclohexanone

\begin{tabular}{ccc}
\hline Temperature $/{ }^{\circ} \mathrm{C}$ & $\mathrm{PS} / \mathrm{ns}$ & $\mathrm{P} \alpha \mathrm{MS} / \mathrm{ns}$ \\
\hline-10 & 15.4 & 18.5 \\
16 & 7.96 & 9.11 \\
26 & 5.91 & 7.21
\end{tabular}

Therefore, we can say from the above finding that the (apparent) dynamic chain stiffness is larger for $\mathrm{P} \alpha \mathrm{MS}$ than for PS.

The present analysis to evaluate the activation energy for the local conformational transition in polymers is successful for the system of PS and P $\alpha$ MS in various solutions. The present analysis also suggests that the local relaxation data in dilute polymer solutions should be treated by taking into account the three independent factors, (A) barrier height inherent to the polymer chain, (B) friction between polymer and solvent, and (C) chain expansion effect, as presented in the introduction of this paper. The effect of factor (B) can be eliminated from the apparent activation energy by use of the Helfand's generalized formula (eq 7), and the effect (B) can be roughly characterized by $A_{1}$ and $A_{2}$.

The results of $E_{\mathrm{a}}$ obtained by such analysis with eq 7, reflecting only the intrinsic barrier height of the polymer chain and chain expansion effect, show higher values in poor solvents than in good solvents. Further, it is revealed from $A_{1}$ and $A_{2}$ that there is no correlation between effects (B) and (C).

Acknowledgment. We are indebted to Dr. Hirokazu Hasegawa for the preparation of the anthracene-labeled P $\alpha$ MS. Present work was supported by a Grant-in-Aid for Scientific Research (No. 62470094) from the Ministry of Education, Science, and Culture of Japan.

\section{REFERENCES}

1. C. Friedrich, F. Laupretre, C. Noël, and L. Monnerie, Macromolecules, 14, 1119 (1981), and papers cited therein.

2. F. D. Blum, B. Durairaj, and A. S. Padmanabhan, Mocromolecules, 17, 2837 (1984).

3. E. Helfand, J. Chem. Phys., 54, 4651 (1971).

4. H. A. Kramers, Physica, 7, 284 (1940).

5. B. Valeur and L. Monnerie, J. Polym. Sci., Polym. Phys. Ed., 14, 11 (1976).

6. T. Sasaki, M. Yamamoto, and Y. Nishijima, Macromolecules, 21, 610 (1988), and papers cited therein.

7. A. T. Bullock, G. G. Cameron, and P. M. Smith, $J$. Chem. Soc., Faraday Trans., 2, 70, 1202 (1974).

8. W. Gronski, T. Schafer, and R. Peter, Polym. Bull., 1, 319 (1979).

9. F. Laupretre, C. Noël, and L. Monnerie, J. Polym. Sci., Polym. Phys. Ed., 15, 2126 (1977).

10. F. Heatley and A. Begum, Polymer, 17, 399 (1976). F. Heatley and B. Wood, Polymer, 19, 1405 (1978).

11. S. Mashimo, Macromolecules, 9, 91 (1976).

12. W. H. Stockmayer, Pure Appl. Chem., 15, 539 (1967).

13. D. Biddle and Nordström, Arkiv Kemi, 32, 359 (1970).

14. D. A. Waldow, B. S. Johnson, P. D. Hyde, M. D. Ediger, T. Kitano, and K. Ito, Macromolecules, 22, 1345 (1989).

15. D. Y. Yoon, P. R. Sundararajan, and P. J. Flory, Macromolecules, 8, 776 (1975).

16. P. R. Sundararajan, Macromolecules, 10, 623(1977). 